University of Nebraska - Lincoln

DigitalCommons@University of Nebraska - Lincoln

Gordon Gallup Publications

Research Papers in Physics and Astronomy

September 2006

\title{
An investigation of electron helicity density in bromocamphor and dibromocamphor as a source of electron circular dichroism
}

\author{
A.M. Scheer \\ University of Nebraska - Lincoln \\ Gordon A. Gallup \\ UNL,ggallup1@unl.edu \\ Timothy J. Gay \\ University of Nebraska - Lincoln, tgay1@unl.edu
}

Follow this and additional works at: https://digitalcommons.unl.edu/physicsgallup

Part of the Physics Commons

Scheer, A.M.; Gallup, Gordon A.; and Gay, Timothy J. , "An investigation of electron helicity density in bromocamphor and dibromocamphor as a source of electron circular dichroism" (2006). Gordon Gallup Publications. 42.

https://digitalcommons.unl.edu/physicsgallup/42

This Article is brought to you for free and open access by the Research Papers in Physics and Astronomy at DigitalCommons@University of Nebraska - Lincoln. It has been accepted for inclusion in Gordon Gallup Publications by an authorized administrator of DigitalCommons@University of Nebraska - Lincoln. 


\title{
An investigation of electron helicity density in bromocamphor and dibromocamphor as a source of electron circular dichroism
}

\author{
A. M. Scheer, G. Gallup and T. J. Gay \\ Behlen Laboratory of Physics, University of Nebraska-Lincoln \\ Correspondence email: tgay1@unl.edu
}

Submitted February 2006; revised March 2006; published April 18, 2006.

\begin{abstract}
We investigate the causes of electron-circular dichroism (ECD) in bromocamphor and dibromocamphor, focusing specifically on the electron helicity density of the target. Using electron transmission spectroscopy (ETS) and quantum chemical calculations, we have observed and assigned temporary negative ion states of bromocamphor and dibromocamphor. Further calculations were conducted to determine the helicity densities of these compounds. Large helicity densities are found in the regions of large wavefunction amplitude of the normally unoccupied molecular orbitals responsible for resonances in the scattering cross sections. We relate our ETS assignments and helicity density results to the chiral asymmetry data observed in electron-circular dichroism experiments by the Münster group (Nolting et al 1997 J. Phys. B: At. Mol. Opt. Phys. 30, 5491). Our results support helicity density as a possible source of chiral asymmetry at certain resonance positions in bromocamphor and dibromocamphor.
\end{abstract}

\section{Introduction}

The cross section for scattering of longitudinally polarized electrons by an enantiomerically pure sample of chiral molecules depends on the chirality of both the target and projectile. Such selectivity is called 'electron-circular dichroism (ECD)', in analogy with its optical equivalent, and has been observed in a variety of molecules by the M"unster group [1-4]. As detailed below, ECD can result from a number of mechanisms, one of which is linked to the electron helicity density [5] of the target molecule. This work studies the implications of electron helicity density of target molecules in the generation of ECD. 


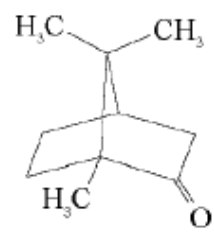

I. Camphor

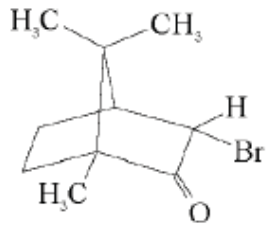

II. 3-Bromocamphor

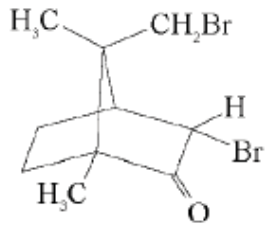

III. 3,9-Dibromocamphor

Figure 1. The structure of the molecules studied here with labels used throughout the text.

In this paper, we present calculated ground state helicity densities of several molecules studied by the Münster group [2, 3], as shown in figure 1: camphor (I), bromocamphor (II) and dibromocamphor (III). We show that in certain important instances these densities have significant spatial overlap with the electronic wavefunctions associated with the lowest few normally unoccupied molecular orbitals responsible for low-energy resonant scattering. In addition, we determine vertical attachment energies (VAEs) associated with lowenergy electron impact on the compounds in figure 1, as measured by electron transmission spectroscopy (ETS) [6] and compare our results with ET spectra reported by Mayer et $a$ l [2]. We also report scaled virtual orbital energies (VOEs) generated by ab initio quantum chemical calculations and show how trends in these values correlate with our experimentally obtained VAEs for this family of molecules.

In the Münster experiment, a longitudinally polarized electron beam of incident intensity Io passes through a chiral vapour target and the intensity of the transmitted beam, $\mathrm{I}^{ \pm}$, is measured when the incident beam is predominantly spin forward (+) or backward (-). From these data, a transmission asymmetry can be constructed:

$$
A=\frac{I^{+}-I^{-}}{I^{+}+I^{-}}
$$

When the handedness of the target is reversed, the asymmetry must change sign. The time-reversed circularly dichroic effect must also exist: unpolarized incident electrons develop longitudinal polarization as they traverse the target, as has been elegantly demonstrated by Nolting et al [3].

The Münster results can be summarized as follows. The asymmetry parameter was measured between $\sim 1 \mathrm{eV}$ and $10 \mathrm{eV}$ for targets of I, II and III, (+)-iodomethylbutane, (+)bromomethylbutane and a series of NMR shift reagent 'propeller molecules': X tris[3(hep tafluoropropylhydroxylmethylene)- $( \pm)$-camphorate] (X(hfc)3), where X Pr, Eu, Er and Yb. The ligands or 'blades' of the NMR propeller molecules are camphor-like, and in each target sample, the chirality of these ligands was pure. Their conformational arrangement about the central, high- $Z$ atom for a given target sample, however, was racemic. The values of are small, generally not exceeding $2 \times 10^{-4}$ except at energies below $1 \mathrm{eV}$ where, in the Er and Pr targets, $A$ approaches $4 \times 10^{-4}$. This latter increase appears to be an artifact associated with an energy-dependent transmission in the apparatus at low energy [3].

One could reasonably expect that would be enhanced in the regions where temporary negative ions are formed, because resonant states might give the target and projectile an enhanced probability of 'sampling each other's chirality' [7, 8]. Indeed, the Münster data exhibit a resonance-like structure which is reminiscent of the resonance structure in the total cross sections in the cases where these latter data were obtained. The correspondence, however, is qualitative at best. In the NMR camphor-lanthanoid complexes, where data on both and the cross section 
exist for the complete series, the fairly compelling correlation between cross section and asymmetry features for the $\mathrm{Yb}$ compound is absent for the other compounds in the series.

Most theories of ECD predict that A should scale as $Z^{2}$, where $\mathrm{Z}$ is the highest nuclear charge in the target [8]. This is observed roughly in the Münster data. Camphor $(\mathbf{I} ;=8)$ exhibits no appreciable effect, while II $(=35)$ and the camphor-lanthanide complexes all have maximum values exceeding $10^{-4}$. This raises the issue, however, of the importance of target stereochemistry. For example, Z-dependence and/or is likely to be small if the high-atom is separated from the molecule's centre of chirality. One might naively expect the $\mathrm{Yb}(\mathrm{hfc}) 3$ target $(=70)$ to produce asymmetries four times that of II, yet they are of similar magnitude. There is no trend in the maximum value of for the camphor-lanthanide sequence as is increased from 59 to 70 , corresponding to an increase in $Z^{2}$ of more than $40 \%$.

Because symmetry permits non-zero values of $A$, the central question becomes what dynamical scattering mechanisms actually cause it to be non-zero. Three qualitatively distinct mechanisms have been proposed [8].

(1) Mott/plural scattering [9]: in this picture, ECD results from a spin-dependent Mott scattering event from the highest-nucleus in the molecule, preceded or followed by pure Coulombic scattering from other atoms in the same molecule. The target's lack of inversion symmetry prevents these sequential events from being averaged over molecular orientations to eliminate a dichroic effect. 'Mott scattering' in this context is taken to mean any spin-orbit coupling with the continuum electron wavefunction. Thus an equivalent, more manifestly quantum-mechanical way of viewing this mechanism is, in analogy with the Fano effect, that the spatial part of the relevant molecular orbitals may be different for different couplings of orbital and spin angular momentum. Therefore, spindependent scattering cross sections may result. The size of the transmission asymmetry resulting from this mechanism (assuming complete incident electron polarization) should be of the order of $\eta(\alpha \mathrm{Z})^{2}$, where $\eta$ is a parameter used to gauge the chirality of the target's stereochemical structure. To set the scale, Hegstrom has estimated that 'twisted ethylene' [10] has an $\eta$ of $10^{-2}$ [5].

(2) Interference between electric and magnetic dipole moments [11, 12]: in a chiral molecule, state-to-state magnetic-and electric-dipole transition amplitudes caused by the electromagnetic pulse of a passing electron can interfere. This results in a transient magnetic moment along the beam axis, independent of the electron polarization. The induced magnetic moment will have a different effect on incident electrons of opposite spin due to spin-other-orbit interactions, resulting in ECD asymmetries of order $\eta \alpha$. There is no explicit dependence on for this effect. The relevant molecular electric and magnetic polarizabilities can depend implicitly on $Z$, but are more strongly correlated with the molecule's atomic weight.

(3) Helicity density dynamics $[5,8]$ : the target electron helicity operator is $\hbar^{-1} \hat{p}$. $\vec{s}$, where $\hat{p}$ is the unit vector in the direction of the electron momentum and $\vec{s}$ is the electron's spin operator. Due to the spin-orbit interaction between the target electrons and a chiral arrangement of the nuclei, the expectation value of this operator, averaged over all of the targets electrons, is non-zero. This can result in a spin asymmetry if, classically speaking, there is a dynamical difference in the scattering of incident electrons by target electrons having velocity components of opposite sign along the beam direction. Such effects should scale as $\eta(\alpha Z)^{2}$. 
(a)
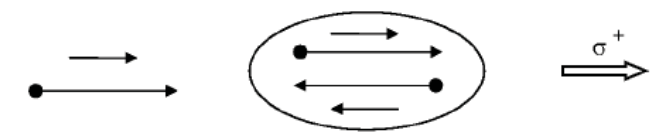

(b)
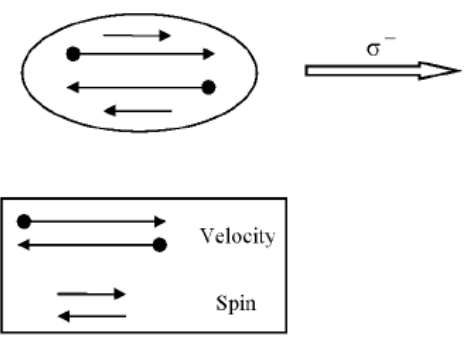

Figure 2. Schematic diagram of polarized electron scattering by a target of a given chirality (see the text). The arrows of different sizes to the right indicate different forward-scattering cross sections for spin-forward (right-handed; +) electrons versus spin-backward (left-handed; -) electrons.

In general, for a given target, all of the above three mechanisms may contribute to the value of $A$. However, for a family of similar molecules it may be possible to isolate a distinct contribution from one mechanism. For instance, one might expect that mechanism (2) is dominant in a series with similar stereochemistry and increasing $Z$, but in which no $Z$-dependence of $\mathrm{A}$ is evident. This is the case with the Münster data for the camphor- lanthanide targets.

In this work, we investigate the helicity-density mechanism as it pertains to the target series of I, II and III. The dynamical mechanism whereby an integrated non-zero value of helicity density in the target can result in a forward scattering asymmetry can be visualized as follows (figure 2). We make the simplifying assumption that the interaction between the chiral nuclear arrangement and the electrons is such that a pure target helicity results, i.e., that the target electrons are all in one helicity state. Thus, in figure 2, if a target electron's velocity is along $-\hat{x}$, then its spin is 'backward', etc. We also assume, for simplicity, that no magnetic spin flips occur. Consider scattering to a specific angle of both (a) spin-forward and (b) spin-backward electrons by the target. The 'direct' and 'exchange' amplitudes for this scattering channel are thus

and

$$
\text { (a) } \begin{aligned}
\overrightarrow{\mathrm{e}}+\overrightarrow{\mathrm{T}} & \rightarrow \overrightarrow{\mathrm{e}}+\overrightarrow{\mathrm{T}} & & f-g \\
\overrightarrow{\mathrm{e}}+\overline{\mathrm{T}} & \rightarrow \overrightarrow{\mathrm{e}}+\overline{\mathrm{T}} & & f^{\prime} \\
& \rightarrow \overline{\mathrm{e}}+\overrightarrow{\mathrm{T}} & & g^{\prime}
\end{aligned}
$$

(b) $\overline{\mathrm{e}}+\overrightarrow{\mathrm{T}} \rightarrow \overline{\mathrm{e}}+\overrightarrow{\mathrm{T}} \quad f$

$$
\begin{array}{rlrl}
\rightarrow & \overrightarrow{\mathrm{e}}+\overline{\mathrm{T}} & g \\
\overline{\mathrm{e}}+\overline{\mathrm{T}} \rightarrow \overline{\mathrm{e}}+\overline{\mathrm{T}} & f^{\prime}-g^{\prime},
\end{array}
$$

where the arrow superscripts refer to the electron spin directions. (We consider, again for simplicity, that the incident electron is scattered by single target electrons.) The primed (unprimed) amplitudes correspond to scattering from target electrons that are moving backwards (forward). These will generally be different due to purely dynamical, Coulombic effects, independent of spin. The forward scattering cross section is then given by

$$
\sigma=\frac{1}{2}\left|f^{\prime}\right|^{2}+\frac{1}{2}\left|g^{\prime}\right|^{2}+\frac{1}{2}|f-g|^{2}
$$


for spin-forward incident electrons and by

$$
\sigma^{\prime}=\frac{1}{2}|f|^{2}+\frac{1}{2}|g|^{2}+\frac{1}{2}\left|f^{\prime}-g^{\prime}\right|^{2}
$$

for spin-backward incident electrons. Since these two cross sections are manifestly different, a non-zero scattering spin asymmetry for any given angle can result. Transmission experiments of the type discussed here involve the detection of electrons in the primary beam that are unscattered. Thus integration over all undetected scattering angles is necessary to determine the value of $A$.

\section{Electron transmission spectra}

At resonance energies, the transmission asymmetry A may be enhanced due to the lengthened time the incident electron spends near the chiral centre of the molecule $[7,8]$. In molecules, resonances often occur when an electron becomes transiently bound in a normally unoccupied molecular orbital, thus forming a temporary negative ion (TNI). To investigate the possible correlations between resonance energies and transmission asymmetry, we examined the TNI states of II and III with the use of ETS [6]. The ET spectrum for I has been measured previously in our laboratory [7]. The Münster group has reported ET spectra for I and II [2].

In our current ETS experiment, a magnetically collimated, monoenergetic electron beam is transmitted through a collision cell containing a target vapour. Scattered electrons are rejected by a retarding electrode following the collision cell and the transmitted electrons are collected. For a given incident energy and energy loss, the amount of retarding voltage determines the electron beam scattering angles that are rejected. To accentuate the resonant structure in the cross section, a small modulation voltage is applied directly to the collision cell and the ac component of the transmitted current is sent to a lock-in amplifier, enabling detection of the derivative of transmitted current with respect to energy. In the derivative signal, a peak in the total scattering cross section is thus indicated by a minimum followed by a maximum. The resonance energy is assigned to the vertical midpoint of this dip-to-peak structure. Because the electron attachment process is rapid relative to nuclear motion, the resonance energy characterizes the molecule in its neutral equilibrium geometry and is a measure of the vertical attachment energy (VAE). A peak at $2.46 \mathrm{eV}$ in the derivative signal of N2 associated with vibrational structure $(v=2)$ of the ${ }^{2} \Pi_{\mathrm{g}}$ anion state is used to provide an energy calibration of the ET spectra in this work as was done in [7]. A trochoidal electron monochromator [13] provided an incident electron energy width between 40 and $80 \mathrm{meV}$. The absolute uncertainty in the determination of resonance energies is $\pm 0.1 \mathrm{eV}$.

Samples II and III had very low vapour pressure at room temperature. These compounds were introduced into the collision cell by means of a sample oven directly attached to the collision chamber. To obtain adequate vapour pressure, II required a temperature of $\sim 60^{\circ} \mathrm{C}$ while III required $\sim 150^{\circ} \mathrm{C}$. The oven and collision cell are separately heated with the cell kept slightly warmer to avoid condensation. Measurements were taken with 10-40\% attenuation of the electron beam. The ET profiles remained unchanged with increasing temperature, indicating no sample decomposition was occurring.

In figure 3, we present the ET spectra for the compounds studied here, plotting the derivative of the transmitted current as a function of incident electron energy. Vertical lines indicate the midpoints of the resonances and the labels give the corresponding empty orbital assignments discussed below. Weaker retarding voltages are employed at higher energies to avoid variations in the transmitted current associated with the alignment of the magnetic field with the electron beam [14]. The breaks in the curves separate spectra obtained with different retarding voltages and different scaling factors. The curves are overlapped to show energy agreement. 


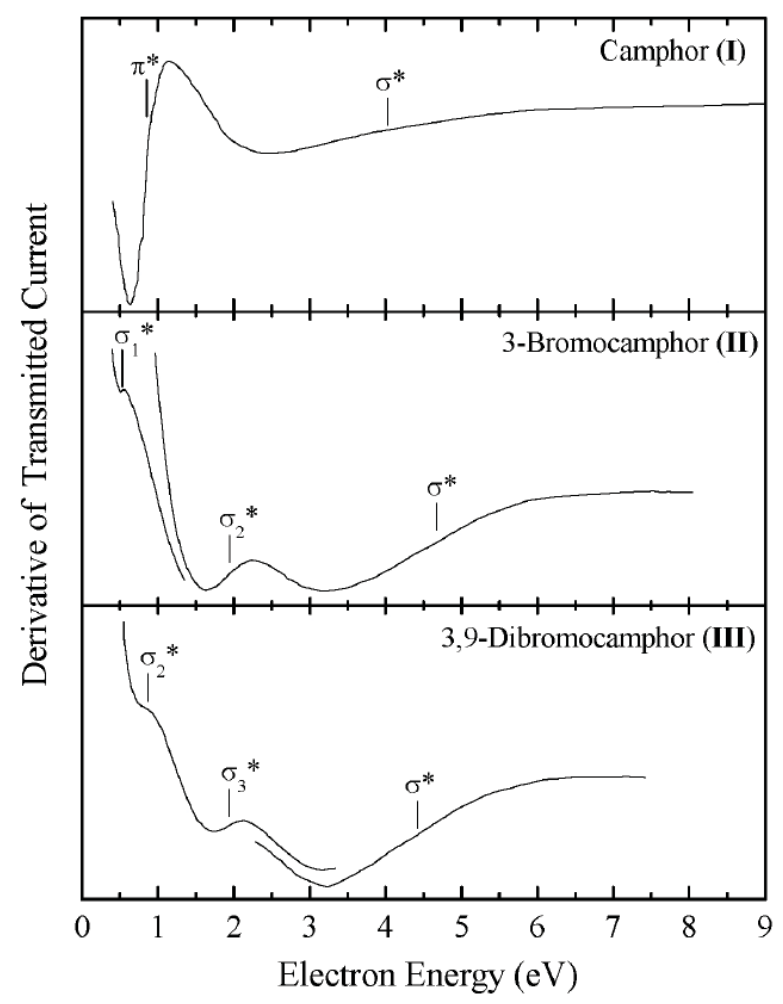

Figure 3. Derivative with respect to electron energy of the current transmitted through gas phase camphor, 3-bromocamphor and 3,9-dibromocamphor. Vertical attachment energies (VAEs) are indicated by short vertical bars and labelled to indicate the orbital responsible for the resonance.

Mayer et al [2] have measured the ET spectra of I and II with a technique similar to ours. We show their comparable data in figures 4(a) and (b). The top panels of figure 4 are the spectrum of from [7] and the spectrum of II from this work. The bottom panels are the corresponding spectra of Mayer et $a \mathrm{l}$ [2]. Vertical lines designating assigned resonance positions in the bottom panels are as given in [2]. The two ET spectra of agree qualitatively and quantitative agreement is observed at low energy. However, the vertical midpoint of the second resonance $\left(\sigma^{*}\right)$ in [7] is seen at $4.0 \mathrm{eV}$, as opposed to $\sim 5.0 \mathrm{eV}$ as observed by the Münster group. A similar difference is observed for the high-energy resonance in II. A more qualitative disagreement is apparent for the low-energy spectra of II. We observe the second of three resonances at an energy of $1.94 \mathrm{eV}$, more than $0.5 \mathrm{eV}$ higher than that reported by Mayer et al. These discrepancies may arise because of our use of a retarding voltage to greatly restrict the forward scattering acceptance angle, thus providing a measurement as close as possible to the total scattering cross section. Mayer et al [2] make no mention of such a procedure. Further details of the effects of the retarding voltage on ET spectra can be found in [14].

\section{Orbital assignments}

As an aid in interpreting our ETS results, geometry optimized $a b$ initio quantum chemical calculations at the 6-31G(d) level were performed on the molecules using the GAMESS program suite [15]. The unfilled orbitals generated by the self-consistent field calculation 


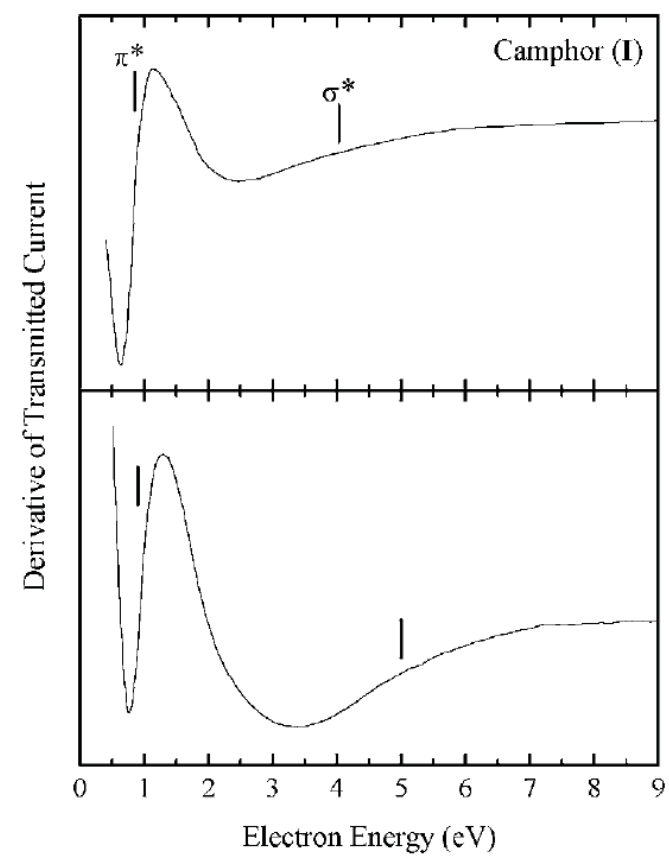

(a)

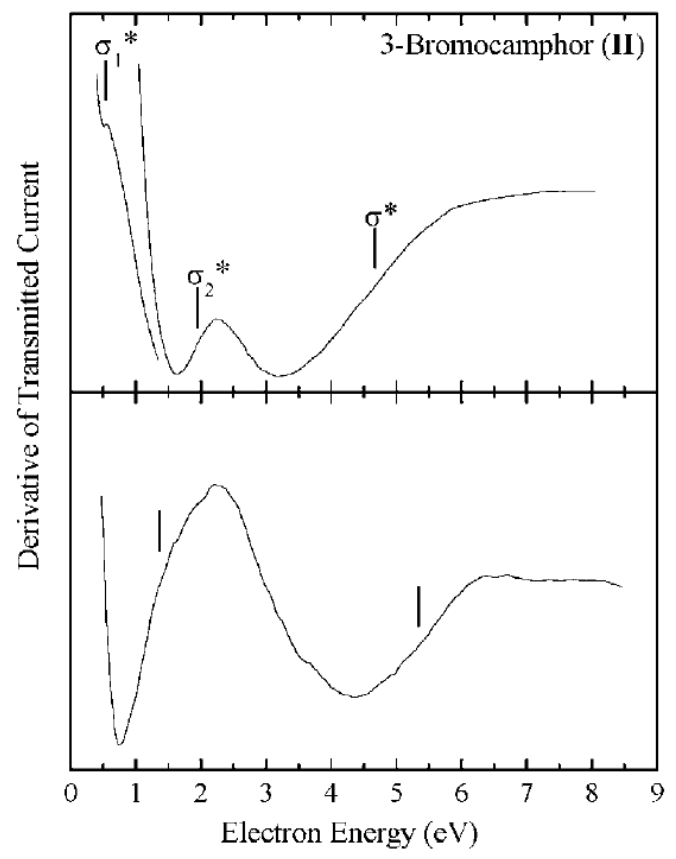

(b)

Figure 4. (a) A comparison of electron transmission spectra for camphor reported in [2] (lower panel) and [7] (upper panel). Vertical lines indicate resonance energies. (b) A comparison of electron transmission spectra for 3-bromocamphor obtained in this work (upper panel) and reported in [2] (lower panel). Vertical lines indicate resonance energies.

are known as virtual orbitals (VOs). The energy of a VO can be used to approximate the energy of the anion resulting from occupation of that orbital according to Koopmans' theorem [16]. The computed energies of the VOs are always too high because interactions with continuum states are not accounted for [17]. Because the VOEs lie just above the vacuum level, their energies are lowered by interaction with continuum states. Another source of error in Koopmans' theorem values is the neglect of electron correlation effects which also results in a lowering of energy [18].

Closer agreement with experimental VAE values may be obtained by using semi-empirical scaling procedures based on the trend between VOEs and VAEs of previously studied molecules as discussed by Chen and Gallup [17] and Staley and Strnad [18]. The predictive value of scaled VOEs is generally a function of the structural similarity of the molecular family being studied to those compounds used to generate the scaling. Such a VOE versus $\mathrm{VAE}$ trend has been established for compounds containing $\mathrm{C}-\mathrm{Cl}$ bonds [19]. We expect the general character of $\mathrm{C}-\mathrm{Br} \sigma$ orbitals to be similar to that of $\mathrm{C}-\mathrm{Cl}$ orbitals. Thus, we employ this trend here as an aid in orbital assignments. Because of the primarily $\pi$ character of the lowest unoccupied molecular orbital (LUMO) of $\mathbf{I}$, the $\pi^{*}$ scaling given in [17] was used.

Table 1 summarizes the results of our calculations along with VAEs observed by ETS. Figures 5 and 6 show the orbitals relevant to the following discussion as generated by the Molekel 3D plotting software [20] using the output of the GAMESS calculations. In I and II, we attribute the lowest energy feature to temporary occupation of the LUMO. In III, the scaled VOE of the LUMO is much lower than the lowest energy fea- 


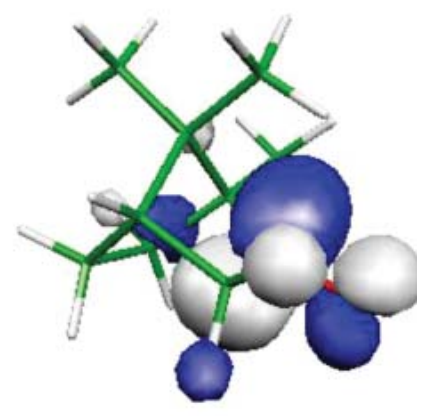

LUMO $\left(x^{*}\right)$

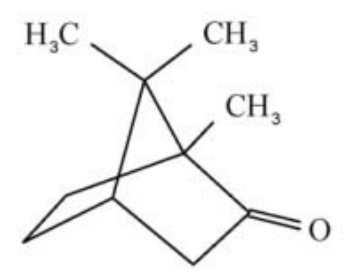

Figure 5. The LUMO of camphor (I). The stick figure structure is included in a similar orientation as a guide to the eye.

Table 1. Vertical attachment energies from the Münster and Nebraska labs along with virtual orbital energies and scaled VOEs from 6-31G(d) basis set calculations. Orbital assignments are given. All energies are reported in $\mathrm{eV}$. The \# indicates that the value was obtained using the $\pi^{*}$ correction [17] (see the text).

\begin{tabular}{llllll}
\hline \multirow{2}{*}{ Compound } & $\begin{array}{l}\text { (Nebraska) } \\
\text { ETS }\end{array}$ & $\begin{array}{l}\text { (Münster) } \\
\text { ETS [2] }\end{array}$ & VOE & $\begin{array}{l}\text { Scaled VOE } \\
\sigma * \mathrm{c}-\mathrm{Cl}\end{array}$ & $\begin{array}{l}\text { Orbital } \\
\text { assignment }\end{array}$ \\
\hline I & 0.87 & 0.90 & 4.53 & $1.68^{\#}$ & LUMO \\
\multirow{4}{*}{ II } & 4.00 & 5.00 & - & - & \\
& 0.53 & - & 3.49 & 0.60 & LUMO \\
& 1.94 & 1.37 & 5.05 & 2.00 & LUMO + 1 \\
\multirow{4}{*}{ III } & 4.68 & 5.34 & - & - & - \\
& - & - & 3.16 & 0.29 & LUMO \\
& 0.95 & - & 4.16 & 1.20 & LUMO + 1 \\
& 1.94 & - & 5.05 & 2.00 & LUMO + 2 \\
& 4.41 & - & - & - & - \\
\hline
\end{tabular}

ture observed in ETS. The first resonance energy observed in III agrees well with the scaled energy of the LUMO + 1 (LUMO + 1 refers to the second lowest unoccupied orbital etc). A second feature with a VAE of $1.94 \mathrm{eV}$ is seen in both II and III. Orbitals with very similar wavefunctions and scaled energy of $2.00 \mathrm{eV}$ are calculated for both compounds. Because of the identical energies and similarity of the features appearing in the total scattering cross section, we feel confident assigning these resonances to the temporary occupation of the LUMO + 1 and the LUMO + 2 for II and III, respectively. In all three compounds, a broad feature centred between 4 and $5 \mathrm{eV}$ is observed. Because these structures span a large energy range and each molecule possesses several higher energy orbitals, it is difficult to attribute any single orbital to this resonance.

In figure 5, one can see that the LUMO of resides almost entirely on the $\mathrm{C}=\mathrm{O}$ double bond, similar to a $\pi$ orbital in a planar molecule. In II and III (figures 6(a) and (b)), the 


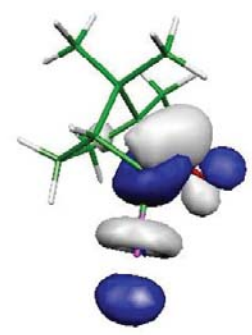

LUMO $\left(\sigma_{1}^{*}\right)$

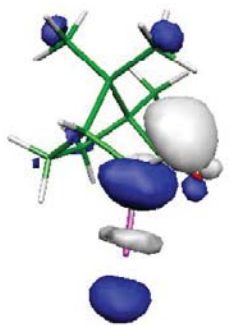

LUMO $+1\left(\sigma_{2}^{*}\right)$

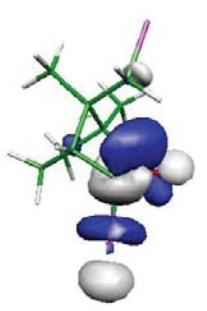

LUMO

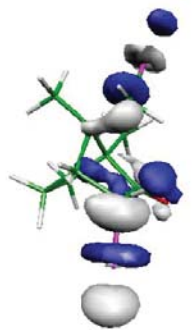

$\mathrm{LUMO}+1\left(\sigma_{2}^{*}\right)$

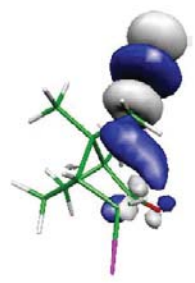

$\mathrm{LUMO}+2\left(\sigma_{3}^{*}\right)$
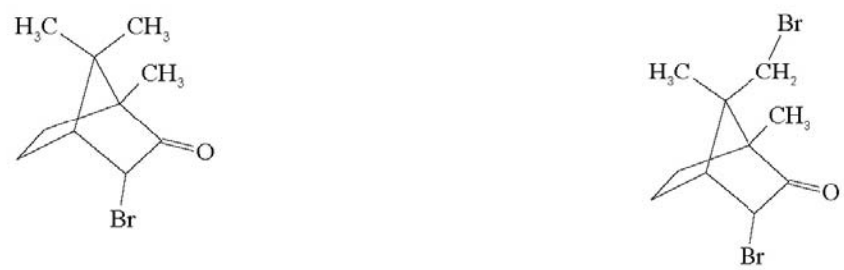

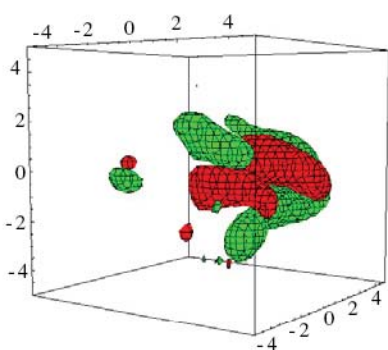

(a)

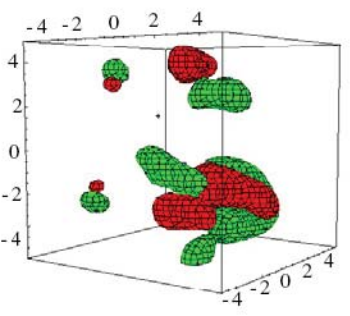

(b)

Figure 6. (a) Top: the lowest few unoccupied orbitals of 3-bromocamphor (II) resulting from geometry optimization calculations at the 6-31G(d) level. Middle: a stick figure structure of II in a similar size and orientation as in the orbital diagrams and helicity density plots. Bottom: contour plots of the calculated helicity density with isosurfaces of 0.28 (units of $\alpha^{2} / 2 \mathrm{bohr}^{-3}$ ). Red lobes indicate regions of positive helicity density while green lobes indicate negative regions. The origin of the plot corresponds to the molecule's centre of mass and numbers along the axes are in units of Angstroms. (b) As in (a) for 3,9-dibromocamphor (III).

wavefunction of the lowest few unoccupied orbitals consists of two primary components. Large lobes exist on the $\mathrm{C}-\mathrm{Br}$ bonds, characteristic of localized $\sigma$ orbitals. Also, to varying degrees, significant wavefunction amplitude, characteristic of $\pi$ orbitals, on the $\mathrm{C}=\mathrm{O}$ bond is present. In the ET spectra of $\mathbf{I}$ in figures 3 and 4, the predominantly character of the LUMO is indicated with a $\pi^{*}$ label, the * signifying that the orbital is antibonding. In II and III, the $\sigma^{*}$ label is employed for the low-energy features. However, one should remain cognizant of the hybrid nature of the orbitals giving rise to these structures. The subscripts on the $\sigma^{*}$ labels indicate the orbital responsible for the respective features (1 corresponds to LUMO, 2 corresponds to LUMO + 1, etc).

The LUMO resonance of II and the LUMO + 1 resonance of III are partially obscured by the electron beam profile. Because the full dip-to-peak structure distinctive of a resonance is not visible, the energies obtained by ETS may not be a measure of the true vertical midpoint. Thus, a strong correlation between our scaled VOEs and these ET energies may not be expected. The lack of agreement between the scaled LUMO energy of (using the correction [17]) with the VAE observed by ETS is puzzling because the resonance is not obscured and is at low energy where good agreement with the scaled VOEs is usually found. 


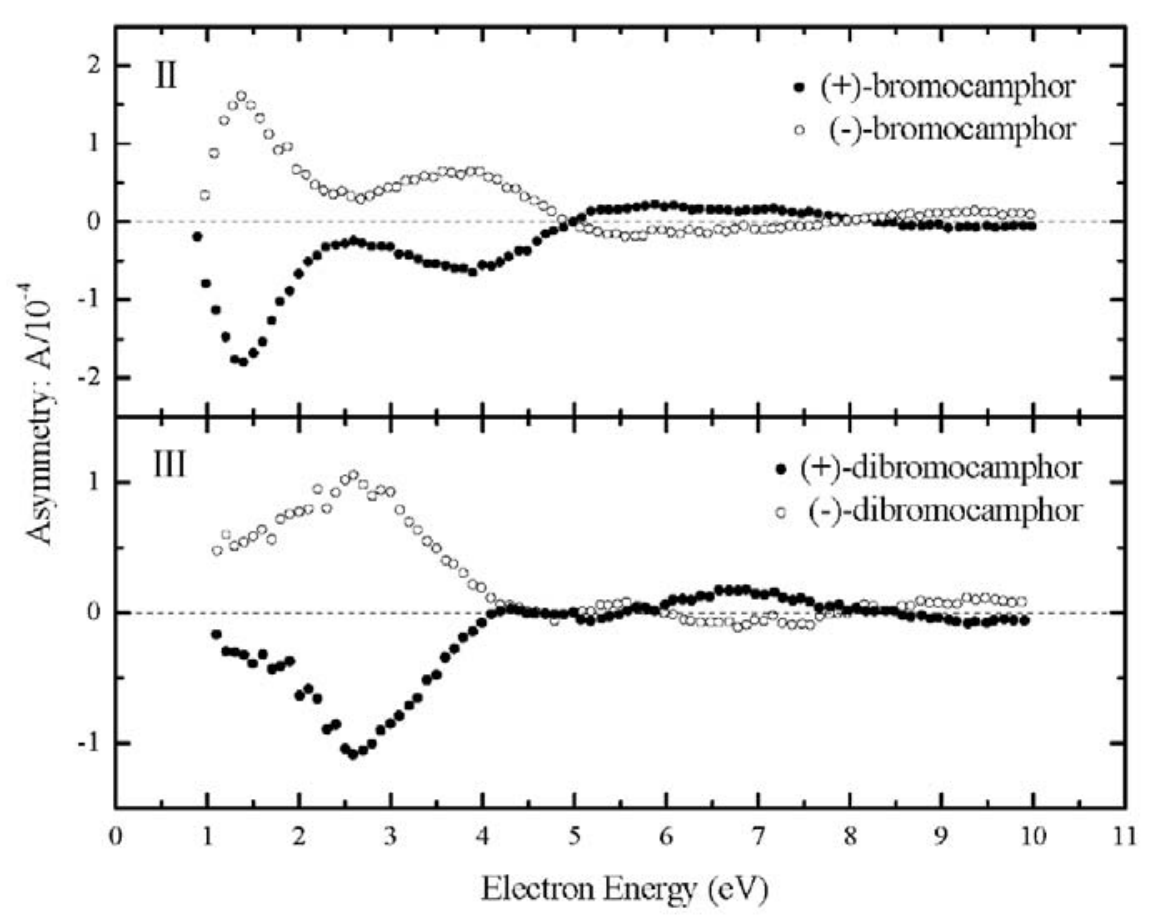

Figure 7. Asymmetry versus incident electron energy of 3-bromocamphor (II) and 3,9-dibromocamphor (III) reproduced from [2]. Error bars are not shown, but are generally comparable to or smaller than the size of the data points above $2.0 \mathrm{eV}$. They increase to $\sim 2 \times 10^{-5}$ below $2.0 \mathrm{eV}$.

\section{Helicity density and implications for ECD}

A significant asymmetry was not observed for $\mathbf{I}$, but as mentioned, asymmetries of greater than $10^{-4}$ were seen in both II and III [3]. The ECD asymmetry data in II, reproduced in figure 7 , show a sharp peak at $\sim 1.4 \mathrm{eV}$ attributed to resonant scattering [3], along with broader features at $\sim 3.8 \mathrm{eV}$ and $\sim 6.0 \mathrm{eV}$. The data for III (figure 7 ) display a shoulder near $1.4 \mathrm{eV}$ that continues into a peak at $\sim 2.6 \mathrm{eV}$. The aforementioned peak in II and shoulder in III appear at nearly the same energy and fairly close to the scaled VOEs $(2.00 \mathrm{eV})$ and identical VAEs $(1.94 \mathrm{eV})$ we observed and assigned to the LUMO + 1 and LUMO + 2, respectively, as listed in table 1. Because of these similarities, we suggest that resonant scattering associated with temporary electron occupation of the LUMO +1 of II and the LUMO + 2 of III is the mechanism by which these particular features in the asymmetry data arise. However, resonant behaviour does not appear to account for the higher energy maxima in A for II. As mentioned above and as can be seen in figure 4(b), Mayer et al [2] observe a resonant state in II centred at $\sim 1.4 \mathrm{eV}$, an energy that correlates better with the asymmetry data. Because neither of the ETS or ECD experiments done at Münster appears to use retarding fields in the target region, the correlation between their resonance positions and asymmetry maxima is consistent, given that neither of their experiments is a measure of the total scattering cross section. The ECD experiments conducted by the Münster group were limited to an energy range above $\sim 1 \mathrm{eV}$. Our observation of temporary negative ion states below this energy in both II and III indicates that obtaining asymmetry data below $1 \mathrm{eV}$ is highly desirable.

To further investigate the resonance dynamics of ECD, we have calculated the helicity density of ground states II and III with the following procedure. Conventional re- 
stricted closed-shell Hartree-Fock (RHF) quantum chemical calculations were performed with GAMESS [15] using a minimal Huzinaga (3G) [21] basis set, labelled as 'MINI' in GAMESS vernacular. The resulting molecular orbitals (MOs) were used in a standard perturbation calculation to find the first-order correction to the singlet wavefunction due to the spin-orbit interaction. This correction is a sum over triplet configurations. It is this spinspoiling mixture that produces helicity density in our model. We use a one-particle form of the spin-orbit operator. In atomic units, this is

$$
H^{\mathrm{SO}}=\frac{\alpha^{2}}{2} \sum_{i}\left(\vec{E}_{i} \times \vec{p}_{i}\right) \cdot \vec{s}_{i},
$$

where $\overrightarrow{E_{i}}, \overrightarrow{p_{i}}$ and $\overrightarrow{s_{i}}$ are the electric field, momentum and spin operators for the $i$ th electron, respectively. Thus, the total wavefunction may be written as

$$
\Psi=\Psi^{(0)}+\Psi^{(1)},
$$

and the correction is a sum over triplet-coupled configurations obtained with single excitations out of a selected set of the occupied MOs into the virtual orbitals.

There are two possible forms of the helicity operator that might be considered. The simpler is the inner product of the electron momentum and the spin, $\rightarrow_{p} \cdot \rightarrow_{s}$. As written, this has dimensions of $M^{2} L^{3} / T^{2}$, which is not particularly transparent, nor is it easily reduced to something more intuitive by multiplying by various physical constants. A dimensionless form for the operator, more in line with photon helicity is $\hbar^{-1} p^{\wedge} \cdot \vec{S}$, where $p^{\wedge}$ is the unit vector in the direction of the momentum. It is only slightly more complicated to calculate for Gaussian orbitals than the first form and has been used to obtain our results for the helicity density. The total integrated helicity densities, $H$, for the (-) forms of the molecules, in units of $\alpha^{2} / 2$, are -0.578 for I, -15.600 for II and -15.395 for III. It is interesting to note that the addition of a second high$\mathrm{Z}$ atom in III does not affect the value of $H$ appreciably. This may be due to the fact that the $\mathrm{C}_{9}$ site is not a chiral centre. The ratios of for II and III compared with I are of the same order as the ratios of $Z^{2}$ for the heaviest atom contained in the respective molecules.

Figure 6(a) shows the molecular orbitals discussed above (computed with a geometry optimization calculation at the 6-31G(d) level) and helicity density for II. Figure 6(b) gives the analogous results for III. Because of the small value of in I, helicity density dynamics are not expected to produce significant asymmetries and the corresponding plot is not shown. As expected, and as can be seen in figures 6(a) and (b), the largest magnitudes of the helicity density are in the regions of the heaviest nuclei, the $\mathrm{Br}$ atoms. Since the electron scattering resonances are also associated with the $\mathrm{C}-\mathrm{Br}$ antibonding orbitals, it is these regions that are expected to contribute predominately to the chiral spin effects. As discussed above, we have assigned TNIs appearing at $1.94 \mathrm{eV}$ in the respective ET spectra to electron capture into the LUMO + 1 of II and the LUMO +2 of III. Features in the asymmetries near this energy are much more pronounced in II than III. A comparison of the respective helicity densities and these orbitals is instructive. In II, the LUMO + 1 has large wavefunction amplitude in regions of large helicity density. There is a corresponding large ECD asymmetry near this resonance energy. However, the $\mathrm{LUMO}+2$ of $\mathbf{I I I}$ is located on the $\mathrm{C}_{9}-\mathrm{Br}$ bond (near the bridge vertex), a region remote from the chiral centre of the molecule and in an area of lower helicity density.

These considerations can be quantified by comparing the integrated product of the ground state helicity densities (figures 6(a) and (b)) and the probability densities of the various unoccupied molecular orbitals (corresponding to the squares of the amplitudes plotted in these same figures). This quantity may be expressed alternatively as the matrix element $\langle i|\mathrm{hd}| i\rangle$, for the $i$ th orbital, where hd is the ground state helicity density. This product, 
in units of $\alpha^{2} / 2 \mathrm{bohr}^{-3}$, is +0.109 for the LUMO +1 of $\mathbf{~ I I}$, and is +0.046 for the LUMO + 2 of III. If helicity density mechanisms depending on resonance formation associated with the LUMO + 1 of II and the LUMO + 2 of III are responsible for the asymmetry observed in the respective ECD data, the ratio of these matrix element values would be expected to be the same as the ratio of the corresponding asymmetries at $\sim 1.4 \mathrm{eV}$. As can be seen in figure 7 , at $1.4 \mathrm{eV}$ the asymmetry in II is 3.1 times that in III whereas the corresponding ratio of matrix elements is 2.4 . The similarity of these values supports a helicity density mechanism as a source of ECD at these resonance positions. However, with asymmetry peaks in regions outside of the energy range of the resonant behaviour observed in the total scattering cross section, the possibility of other mechanisms cannot be eliminated.

Peaks at higher energy in the asymmetry data do not correlate with any distinct resonance features in ETS, although helicity density mechanisms cannot be excluded as an explanation for these features. Some asymmetry peaks at higher energy change sign. In the helicity density model, this would correspond to higher energy resonance states having greater overlap with regions of negative ground state helicity density. This is also the case at lower energy with the LUMO +1 resonance in III (with a matrix element of -0.129 ), which may contribute to the relatively low values of $A$ below $2 \mathrm{eV}$ for this molecule.

\section{Conclusion}

We have come to the following conclusions regarding our ETS data, in combination with the ETS and asymmetry data of the Münster group. In camphor (I), the resonance at $\sim 0.87$ $\mathrm{eV}$, observed by both the Münster and Nebraska groups, is due to the LUMO of $\pi$ character. However, this strong resonance does not produce a corresponding ECD effect, presumably because it is not associated with a high- $Z$ atom. The corresponding helicity density at the camphor chiral centre is low. In bromocamphor (II), the feature at $0.53 \mathrm{eV}$, observed only in this work, is due to a LUMO resonance of mixed $\sigma / \pi$ character, while the resonance observed between 1.5 and $2.0 \mathrm{eV}$ unambiguously corresponds to the LUMO +1 . This latter orbital is spatially coincident with a region of large helicity density. The assignment of the prominent asymmetry maximum in II at $1.4 \mathrm{eV}$ to a resonant helicity density mechanism is thus reasonable. No similar conclusions can be safely drawn about the feature in the asymmetry data for II above $3 \mathrm{eV}$. In dibromocamphor (III), we have observed ETS features at 0.95 and $1.94 \mathrm{eV}$ corresponding to LUMO +1 and LUMO + 2 resonances, respectively. The ET spectrum of III was not obtained by the Münster group. The shoulder near $1.4 \mathrm{eV}$ in the asymmetry data of III may be due to a helicity density mechanism. For III, the lower values of in this region, compared with those for II, can reasonably be attributed to the diminished spatial overlap between the orbital responsible for resonant scattering and the target ground state helicity density.

These studies represent a first attempt to consider the specific implications of a helicity density model for the complex dynamics of asymmetry production in chiral electron-molecule scattering.

\section{Acknowledgments}

The authors are indebted to Paul Burrow for use of the electron transmission spectroscopy apparatus, and to Josh Machacek, John Furst and Kayvan Aflatooni for useful discussions and aid in computer graphics. This work was supported by the NSF under grant PHY-0354946. 


\section{References}

[1] Mayer S and Kessler J 1995 Phys. Rev. Lett. 744803

[2] Mayer S, Nolting C and Kessler J 1996 J. Phys. B: At. Mol. Opt. Phys. 293497

[3] Nolting C, Mayer S and Kessler J 1997 J. Phys. B: At. Mol. Opt. Phys. 305491

[4] Zimnol A H, Meyer T, Hamelbeck V and Hanne G F 2005 Book of Abstracts, XXIV ICPEAC

[5] Rich A, Van House J and Hegstrom R A 1982 Phys. Rev. Lett. 481341

[6] Sanche L and Schulz G J 1972 Phys. Rev. A 51672

[7] Stephen T M, Shi X and Burrow P D 1988 J. Phys. B: At. Mol. Opt. Phys. 21169

[8] Gay T J, Johnston M E, Trantham K W and Gallup G A 1996 Selected Topics in Electron Physics ed D M Campbell and H Klempoppen (New York: Plenum)

[9] Kessler J 1982 J. Phys. B: At. Mol. Phys. 15101

[10] Hegstrom R A, Rein D W and Sandars P G H 1980 J. Chem. Phys. 732329

[11] Walker D W 1982 J. Phys. B: At. Mol. Phys. 15289

[12] Gallup G A 1994 Electron Collisions with Molecules, Clusters, and Surfaces ed H Ehrhardt and L A Morgan (New York: Plenum)

[13] Stamatovic A and Schulz G J 1970 Rev. Sci. Instrum. 41423

[14] Johnston A R and Burrow P D 1982 J. Electron Spectrosc. Relat. Phenom. 52119

[15] Schmidt M W et al 1993 J. Comput. Chem. 141347

[16] Koopmans T 1933 Physica 1105

[17] Chen D and Gallup G A 1990 J. Chem. Phys. 9312

[18] Staley S W and Strnad J T 1994 J. Phys. Chem. 98116

[19] Aflatooni K, Gallup G A and Burrow P D 2000 J. Phys. Chem. A 1047359

[20] Portmann S 2000 CHIMIA 54766 see Molekel http://www.cscs.ch/molekel/

[21] Huzinaga S, Andzelm J, Klobukowski M, Radzio-Andzelm E, Sakai Y and Tatewaki H 1984 Gaussian Basis Sets for Molecular Calculations (Amsterdam: Elsevier) 